
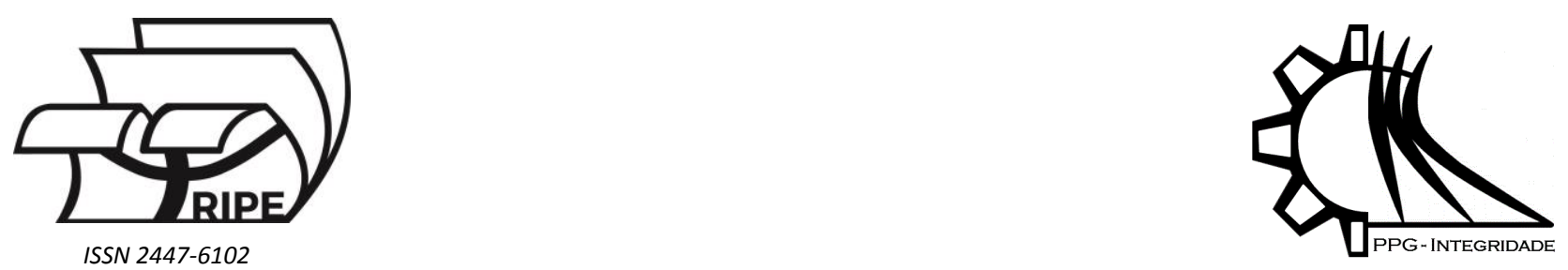

Article

\title{
Estudo numérico do processo de Infusão de Resina Líquida (LRI) utilizando malha de fluxo
}

\author{
Dutra, C.M.B. ${ }^{1}$, Trindade, R.S. ${ }^{2}$, Nunes, S.G. ${ }^{2}$, Amico, S.C. ${ }^{2}$ and Souza, J.A. ${ }^{1, *}$ \\ 1 Universidade Federal do Rio Grande - FURG, Rio Grande - RS, 96203-900, Brasil; carlabulsing.cb@gmail.com \\ 2 Universidade Federal do Rio Grande do Sul - UFRGS, Porto Alegre - RS, 91509-900, Brasil; rafastrindade@gmail.com; \\ stephaniegnunes@hotmail.com; amico@ufrgs.br \\ * correspondente: jasouza@furg.br
}

Received: 14/12/2018; Accepted: 05/01/2019; Published: 16/01/2019

Resumo: O presente trabalho é dedicado a estudar numericamente problemas envolvendo o processo de Infusão de Resina Líquida (LRI) em compósitos espessos. Esta técnica se utiliza de fibras para obtenção de compósitos poliméricos que podem ser aplicados em diversos ramos das indústrias e da engenharia. Nesse sentido, há um grande interesse em aprimorar este processo para obter um produto final com propriedades físicas e mecânicas que atendam às exigências do mercado atual. Esse estudo tem por objetivo analisar o escoamento da resina dentro do molde e otimizar a geometria, a partir do comprimento da malha de fluxo e a relação entre as permeabilidades $K_{x x} / K_{z z}$. A malha de fluxo é um tecido de alta permeabilidade e é empregada durante o processo de moldagem por LRI para facilitar a infiltração da resina através do meio fibroso. As simulações numéricas foram realizadas no software livre OpenFOAM e a criação da geometria e da malha no GMSH. A formulação de volumes finitos do OpenFOAM foi utilizada para a discretização das equações de transporte que descrevem o escoamento de resina dentro do molde e o tratamento da interface entre os dois fluidos (resina-ar) é resolvido pelo Método Volume of Fluid (VOF). Neste artigo, apresentou-se resultados para uma relação de $K_{x x} / K_{z z}$ e diferentes comprimentos de malha de fluxo. Por meio dos resultados apresentados, pode-se encontrar uma maneira de generalizar o estudo, e assim, obter o tamanho ideal da malha de fluxo para qualquer comprimento de molde.

Palavras-chave: Infusão de Resina Líquida; Malha de fluxo; Compósito; Simulação numérica; OpenFOAM.

\section{Numerical study of the Liquid Resin Infusion process (LRI) using flow mesh}

Abstract: The present work is dedicated to numerically study problems involving the Liquid Resin Infusion (LRI) process in thick composites. This technique uses fibers to obtain polymeric composites that can be applied in various lines of the industry and engineering. In this way, there is a great interest in improving this process to obtain a final product with physical and mechanical properties that meet the requirements of the current market. This study aims to analyze the flow of the resin inside the mold and to optimize the geometry, from the length of the flux mesh and the relationship between the permeabilities $K_{x x} / K_{z z}$. The flow mesh is a high permeability fabric and is employed during the LRI molding process to facilitate infiltration of the resin through the fibrous area. Numerical simulations were performed in OpenFOAM free software and the creation of the geometry and the mesh in the GMSH. The finite volume formulation of OpenFOAM was used for the discretization of the transport equations describing the resin flow inside the mold and the treatment of the interface between the two fluids (resin-air) is solved by the Volume of Fluid (VOF) method. In this paper, results for a $K_{x x} / K_{z z}$ ratio and different flow mesh lengths were presented. By the presented results, one can find a way to generalize the study, and thus, to obtain the ideal size of the flow mesh for any length of mold.

Keywords: Liquid Resin Infusion; Flow mesh; Composite; Numerical simulation; OpenFOAM. 


\section{Introdução}

Os compósitos poliméricos, constituídos de fibras vegetais ou sintéticas, estão sendo empregados na área da engenharia desde o século $X X$, sendo que apenas a partir da década de 40 estes começaram a ser utilizados mais acentuadamente em diversos segmentos da indústria de bens de consumo, como os aeronáuticos, automotivos e náuticos, estruturas marítimas, tubulações e ainda em equipamentos militares e esportivos (Gutiérrez et al., 2014). Dessa maneira, a indústria e o meio científico estão procurando aprimorar as propriedades físicas e mecânicas dos materiais para que os produtos possam ser mais leves, resistentes, com uma melhor performance mecânica, além da possibilidade de minimizar os custos de fabricação para atender as exigências do mercado atual.

Diversas técnicas para a produção de materiais compósitos foram desenvolvidas ao longo dos anos e entre elas pode-se destacar a Moldagem por Transferência de Resina (RTM - Resin Transfer Molding) e a Infusão de Resina Líquida (LRI - Liquid Resin Infusion) que será dada ênfase neste estudo.

A infusão líquida de resina, segundo Correia et al. (2005) é um processo que utiliza um molde fechado e apresenta similaridades com o RTM, entretanto a diferença mais marcante é que os moldes rígidos de duas partes utilizados no RTM são substituídos por um molde rígido que é selado com um saco de vácuo no processamento por LRI. Na técnica LRI, simplificadamente, o reforço fibroso é depositado dentro do molde rígido, onde pode-se adicionar uma malha de fluxo que é um tecido de alta permeabilidade e quando adicionado ao processo de moldagem, auxilia na distribuição da resina dentro do molde e diminui o tempo de infiltração. Assim, a bolsa de vácuo é selada para que posteriormente, a pré-forma seja comprimida e ocorra a infusão da resina.

Para assegurar um nível de qualidade mecânica utilizando essa metodologia é necessário que se tenha um cuidado para que vazios na matriz do compósito sejam evitados. Outro fator desafiador é o tamanho do produto, pois embora o ensacamento a vácuo faça uso da pressão atmosférica e permita que o molde possua tamanho grande, há como contrapartida a questão da resina que dependendo da sua viscosidade e o tempo de cura pode restringir esse tamanho (Brouwer, Herpet, \& Labordus, 2003). Portanto, é necessário modelar o processo para reduzir os efeitos e resíduos indesejados, aumentar a umidade e a qualidade da peça ou encurtar o tempo de ciclo (Yalcinkaya, \& Sozer, 2014).

Nesse sentido, diversas pesquisas foram desenvolvidas nos últimos anos como, a de Wang et al. (2011) que desenvolveram um modelo numérico isotérmico, realizando uma comparação entre as simulações numéricas e dados experimentais da infusão em uma placa utilizando o processo LRI. A partir dos resultados numéricos, o tempo de enchimento, a massa de resina e a espessura da pré-forma puderam ser determinados. Os autores salientaram que uma questão importante da pesquisa é destacar as mudanças nos parâmetros do processo, como a temperatura da pré-forma e da resina, variações na espessura e da fração volumétrica da fibra. Hurtado et al. (2015) apresentaram uma análise do escoamento da resina através de um laminado de camadas de fibras de vidro com diferentes orientações. O modelo numérico desenvolvido permite realizar as simulações considerando cada camada como um meio poroso separado, levando em consideração a sequência de empilhamento. $O$ estudo também analisa a influência dos valores de permeabilidade da malha de fluxo e do laminado de fibra de vidro no escoamento da resina.

Magalhães (2016) desenvolveu uma pesquisa realizando uma investigação numérica do processo de infusão de resina aplicando o Design Construtal. Em seu estudo adicionou ao modelo numérico um canal aberto na forma de "I" e " $\mathrm{T}$ " que facilita o escoamento da resina e analisou o efeito da geometria sobre o tempo final de injeção. Loudad et al. (2017) realizaram um estudo que leva em consideração o acoplamento hidromecânico e a coexistência de fluxos planares e transversais, assim a modelagem numérica proposta, permite a simulação do processo de infusão no caso de pré-formas multicamadas com diferentes materiais e orientações, incluindo a malha de fluxo. O modelo proposto foi validado experimentalmente com base em várias infusões.

Este artigo tem por objetivo analisar numericamente o escoamento da resina em moldes de compósitos espessos no processo LRI, bem como otimizar o tamanho da geometria a partir do comprimento da malha de fluxo, esta que é adicionada ao processo pra facilitar o escoamento através do meio fibroso e diminuir o tempo de infiltração. Assim, quando a malha de fluxo possui o mesmo comprimento do molde pode ocorrer da resina que escoa através da malha de fluxo chegue na saída (outlet) primeiro que a resina que escoa na parte inferior do molde, propiciando que não haja a absoluta impregnação das fibras. Portanto, torna-se necessário que se obtenha a projeção do comprimento da malha de fluxo para que a resina chegue ao final do molde de forma linear.

\section{Modelo matemático}

O modelo matemático que descreve o escoamento da resina no interior do molde é fundamentado na Lei de Darcy, expressa por: 


$$
\vec{V}=-\frac{\bar{K}}{\mu} \nabla \mathrm{P}
$$

onde, $\vec{V}$ é a velocidade de escoamento da resina [m/s], $\mu$ é a viscosidade do fluido [Pa s], $P$ é a pressão [Pa], $\bar{K}$ tensor de permeabilidades do meio poroso $\left[\mathrm{m}^{2}\right]$.

Para solucionar as equações que descrevem o escoamento da resina em problemas LRI com o software OpenFOAM, utilizou-se o método do Volume de Fluido (VOF - Volume of Fluid) que modela problemas de escoamento multifásicos e imiscíveis (Hirt, \& Nichols, 1981). As equações de transporte mencionadas são a da conservação da massa, fração volumétrica e quantidade de movimento que serão discretizadas e aproximadas pelo método dos Volumes Finitos e estão expressas respectivamente pelas Eqs. (2), (3) e (4).

$$
\frac{\partial \rho}{\partial t}+\nabla \cdot(\rho \vec{V})=0
$$

onde, $\rho$ é a massa específica $\left[\mathrm{kg} / \mathrm{m}^{3}\right]$ e $t$ o tempo $[\mathrm{s}]$.

$$
\frac{\partial(\rho f)}{\partial t}+\nabla \cdot(\rho f \vec{V})=0
$$

onde, $f$ é a fração volumétrica da resina.

$$
\frac{\partial(\rho \vec{V})}{\partial t}+\nabla \cdot(\rho \vec{V} \vec{V})=-\nabla P+\nabla \overline{\bar{\tau}}+\rho \vec{g}+\vec{F}
$$

em que, $\vec{g}$ é a aceleração da gravidade $\left[\mathrm{m} / \mathrm{s}^{2}\right], \vec{F}$ o termo fonte ou forças externas $\left[\mathrm{N} / \mathrm{m}^{3}\right]$ e $\overline{\bar{\tau}}[\mathrm{Pa}]$ o tensor de tensões. O termo $\vec{F}$ na Eq. (4) é empregado em problemas de meios porosos para incluir um termo de força resistiva na equação do movimento. Nas simulações em que utiliza-se canais abertos, ou seja, sem meio poroso, o termo $\vec{F}$ da Eq. (4) é considerado nulo.

Por fim, como a metodologia VOF resolve as Eq. (2) e (4) para a mistura (neste caso resina + ar), é necessário ponderar as propriedades físicas do fluido (Srinivasan, Salazar, \& Saito, 2011). Desta maneira, tem-se que:

$$
\begin{aligned}
& \rho=f \rho_{\text {resina }}+(1-f) \rho_{a r} \\
& \mu=f \mu_{\text {resina }}+(1-f) \mu_{a r}
\end{aligned}
$$

\section{Domínio computacional e configurações numéricas}

$O$ estudo numérico deste trabalho é dedicado aos problemas LRI em moldes retilíneos. A geometria do problema e as condições de contorno estão de acordo com a Fig. 1, onde, $P$ é a pressão, $u$ e $v$ são as velocidades, $f$ é a fração volumétrica e $n$ a direção normal a superfície. $O$ comprimento do molde corresponde a variável $L_{m}=0,3 \mathrm{~m}$, a altura do meio poroso é $h=0,012 \mathrm{~m}, 11$ e 12 são linhas para monitorar o escoamento da resina na parte inferior e superior do molde e $x(t)$ é a posição da resina quando forma-se a linha de frente do escoamento paralela à saída do molde. A malha de fluxo é considerada nas simulações numéricas como um canal aberto, pois possui uma permeabilidade muito elevada. As dimensões da malha de fluxo estão destacadas na imagem, cuja altura $h_{p}=0,0014 \mathrm{~m}$ e o comprimento $L_{p}$, sendo um valor variável nas simulações. 


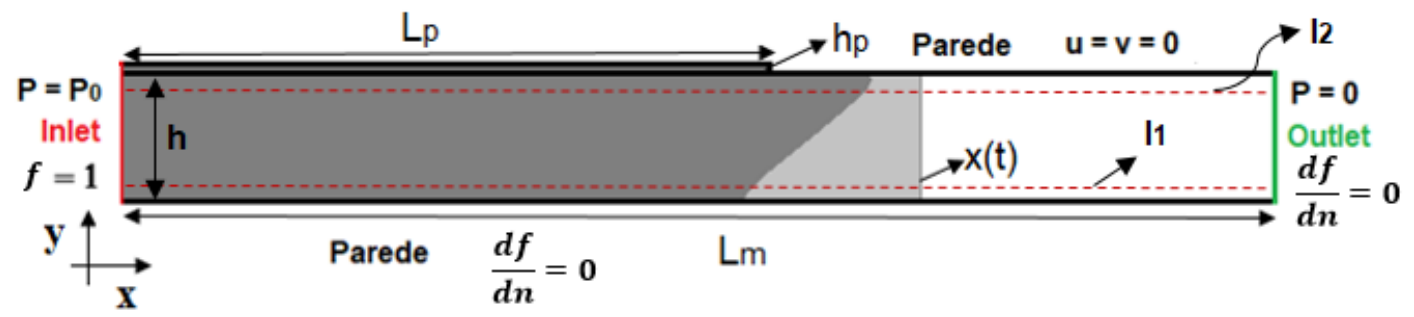

Figura 1. Domínio computacional e condições de contorno.

A geometria utilizada nas simulações corresponde a um modelo 2D e foi construída no software GMSH, bem como a geração da malha. Para identificar a malha ideal para solucionar o problema proposto, realizou-se um teste de independência de malha em que as propriedades utilizadas nas simulações podem ser observadas na Tab. 1 e foram disponibilizadas pelo Laboratório de Materiais Poliméricos (LaPol) da Universidade Federal do Rio Grande do Sul (UFRGS).

Tabela 1. Valores das propriedades utilizada no teste de independência de malha.

\begin{tabular}{ccccc}
\hline$K_{x x}=K_{y y}\left(\mathrm{~m}^{2}\right)$ & $K_{z z}\left(\mathrm{~m}^{2}\right)$ & $\mu(\mathrm{Pa} s)$ & $\varepsilon\left(\times 10^{-2}\right)$ & $P_{0}\left(\times 10^{5} \mathrm{~Pa}\right)$ \\
\hline $2,5 \times 10^{-11}$ & $3,78 \times 10^{-13}$ & 0,3 & 40 & 1 \\
\hline
\end{tabular}

O estudo de refinamento de malha realizado, utilizou-se o tempo final de preenchimento do molde como parâmetro de comparação e considerou-se que a malha ideal seria obtida quando a diferença entre o tempo final de infiltração fosse inferior à $1 \%$. Na Tab. 2 pode-se observar que foram necessários apenas quatro casos para identificar a malha ideal.

Tabela 2. Teste de independência de malha.

\begin{tabular}{cccc}
\hline Caso & № de volumes & Tempo final (s) & Diferença \\
\hline 1 & 4641 & 871 & - \\
2 & 10707 & 924 & $5,74 \%$ \\
3 & 14661 & 937 & $1,39 \%$ \\
4 & 17262 & 943 & $0,64 \%$ \\
\hline
\end{tabular}

Assim, a malha utilizada nas simulações obtém 17262 volumes, constituída de elementos quadrangulares e poder ser observada na Fig. 2. Convém destacar que refinou-se a malha na direção $y$, apenas no canal aberto, pois possui uma altura pequena.

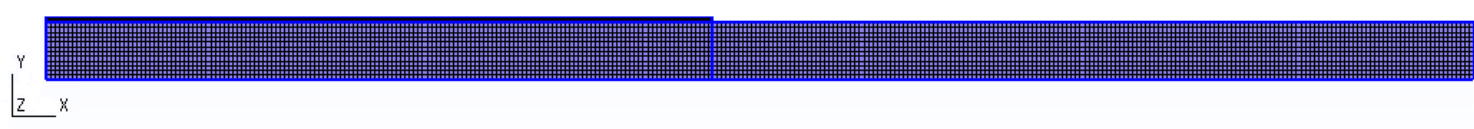

Figura 2. Geometria e malha construídas no GMSH.

Em todas as simulações, para o avanço temporal das soluções, utilizou-se o número de Courant Number, expresso por:

$$
C=\frac{v \Delta t}{\Delta x}
$$

onde $v$ é a velocidade, $\Delta x$ é o comprimento característico do volume e $\Delta t$ é o passo de tempo. A Eq. (7), controla $\Delta t$ para que a solução se mantenha estável, sem ultrapassar o $C$ máximo definido na simulação, que neste caso foi de 
0,5 . Vale destacar, que um $\Delta t$ máximo e um $\Delta t$ mínimo também é definido e foram estipulados com o valor de $1 \times 10^{-3} \mathrm{e}$ $1 \times 10^{-6}$, respectivamente.

As funções de interpolação utilizadas para resolver o problema proposto nesse estudo podem ser observadas na Tab. 3.

Tabela 3. Funções de interpolação utilizadas nas simulações.

\begin{tabular}{cc}
\hline Termo & Função \\
\hline Derivada & Euler \\
Divergente & Gauss linearUpwind \\
Gradiente & Gauss linear \\
Laplaciano & Gauss linear corrected \\
\hline
\end{tabular}

O algoritmo para resolver o problema de acoplamento de pressão-velocidade foi o PIMPLE, este que é uma combinação dos algoritmos SIMPLE e PISO.

\section{Resultados e discussões}

Para analisar o escoamento da resina dentro do molde e otimizar a geometria do problema, ou seja, obter o comprimento ideal do molde para cada valor de $L_{p}$ e relação $K_{x x} / K_{z z}$ inicialmente, investiga-se se a linha de frente do escoamento da resina forma-se na mesma posição $x(t)$ quando varia-se os valores das permeabilidades planares $\left(K_{x x}\right.$ $\left.=K_{y y}\right)$ e a transversal $\left(K_{z z}\right)$, mantendo-se constante a proporção $K_{x \chi} / K_{z z}$.

Nesse sentido, realizou-se cinco simulações cujas propriedades utilizadas estão expressas na Tab. 4 e convém destacar que todas as informações, exceto os valores de permeabilidades, foram disponibilizadas pelo LaPol.

Tabela 4. Valores das propriedades utilizadas nas simulações.

\begin{tabular}{|c|c|c|c|c|c|c|c|}
\hline Simulação & $K_{x x}=K_{y y}\left(\mathrm{~m}^{2}\right)$ & $K_{z z}\left(\mathrm{~m}^{2}\right)$ & $K_{x x} / K_{z z}$ & $\mu(\mathrm{Pa} s)$ & $\varepsilon\left(\times 10^{-2}\right)$ & $P_{0}$ & $\left(x 10^{5} \mathrm{~Pa}\right)$ \\
\hline Caso 1 & $1 \times 10^{-10}$ & $1 \times 10^{-11}$ & 10 & 0,3 & 40 & & 1 \\
\hline Caso 2 & $1 \times 10^{-12}$ & $1 \times 10^{-13}$ & 10 & 0,3 & 40 & & 1 \\
\hline Caso 3 & $3 \times 10^{-10}$ & $3 \times 10^{-11}$ & 10 & 0,3 & 40 & & 1 \\
\hline Caso 4 & $3 \times 10^{-11}$ & $3 \times 10^{-12}$ & 10 & 0,3 & 40 & & 1 \\
\hline Caso 5 & $3 \times 10^{-9}$ & $3 \times 10^{-10}$ & 10 & 0,3 & 40 & & 1 \\
\hline
\end{tabular}

Para identificar a posição $x(t)$ em que forma-se a linha de frente de escoamento linear, estabeleceu-se na simulação duas linhas (/1 e /2) de monitoramento paralelas ao eixo $x$ e que passam pelas coordenadas no eixo $y$ em 0,004 e 0,0115, respectivamente. Considerou-se que a linha de frente de escoamento estava com a frente de onda linear antes da saída do domínio poroso quando obteve-se uma diferença entre a posição da resina sobre a linha superior em relação a linha inferior menor ou igual de 0,0015 m.

$\mathrm{Na}$ Tab. 5 é possível analisar a posição $x(t)$ em que formou-se a linha de frente de escoamento nos cinco casos simulados. Também, apresenta-se as diferenças das posições $x(t)$ das linhas 11 e 12 das simulações nos casos 2,3 , 4 e 5 em relação ao caso 1 , bem como o tempo físico total de preenchimento do molde. 
Tabela 5. Resultados das simulações dos casos investigados.

\begin{tabular}{cccccc}
\hline Simulações & $\begin{array}{c}t(s) \text { de } \\
\text { preenchimento }\end{array}$ & $\begin{array}{c}x(t) \\
\text { Linha 1-I1 }\end{array}$ & $\begin{array}{c}x(t) \\
\text { Linha 2-I2 }\end{array}$ & $\begin{array}{c}\text { Diferença } \\
\text { Linha 1 - I1 }\end{array}$ & $\begin{array}{c}\text { Diferença } \\
\text { Linha 2 - I2 }\end{array}$ \\
\hline Caso 1 & 55 & 0,2669403 & 0,2684452 & - & - \\
Caso 2 & 5320 & 0,2659146 & 0,2674183 & $0,38 \%$ & $0,38 \%$ \\
Caso 3 & 21 & 0,2670909 & 0,2686006 & $-0,06 \%$ & $-0,06 \%$ \\
Caso 4 & 176 & 0,2731734 & 0,2746833 & $-2,34 \%$ & $-2,32 \%$ \\
Caso 5 & 4,6 & 0,2667169 & 0,268224 & $0,08 \%$ & $0,08 \%$ \\
\hline
\end{tabular}

Observa-se que o $x(t)$ encontrado nas simulações realizadas foram muito próximos, evidenciando que é possível utilizar no processo de otimização da geometria apenas valores de permeabilidades mais elevados, desde que a relação $K_{x x} / K_{z z}$ seja mantida. Isso também pode ser analisado na Fig. 3 e 4, onde é possível visualizar as curvas das posições em função do tempo para os casos 1 e 5, respectivamente.

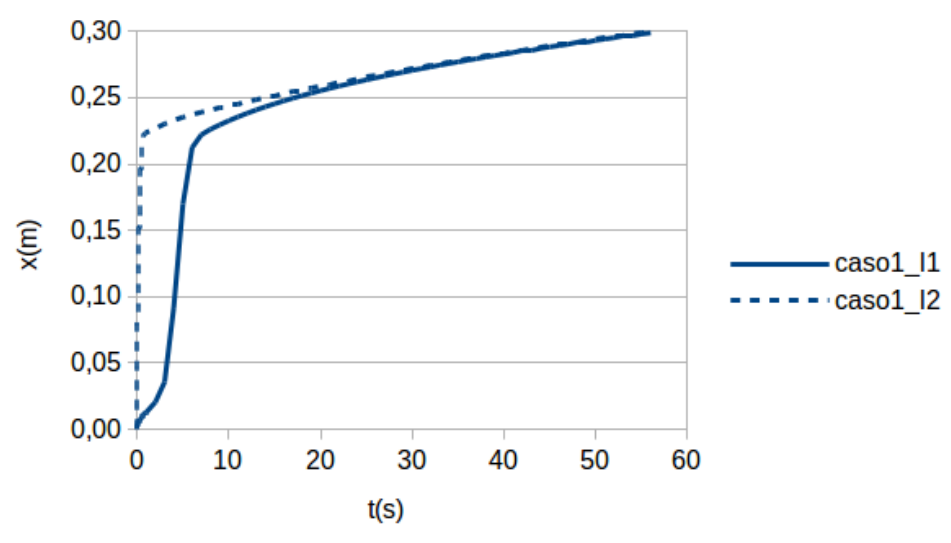

Figura 3. Posição da resina em função do tempo das linhas $/ 1$ e $/ 2$ no caso 1.

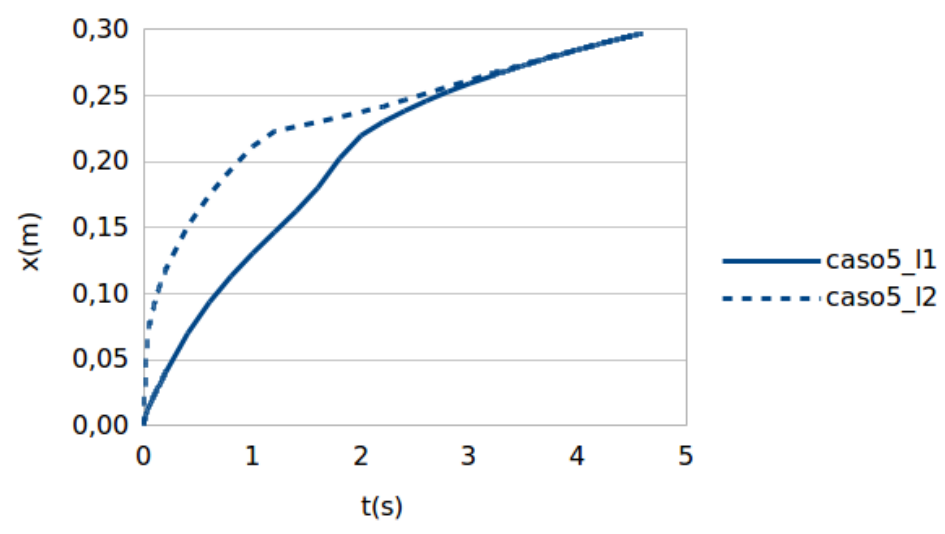

Figura 4. Curvas do escoamento da resina nas linhas $I 1$ e $/ 2$ no caso 5.

A diferença obtida entre as posições $x(t)$ em que formou-se a linha de frente de escoamento entre casos 1 e 5 foi inferior à $0,1 \%$. Além disso, a simulação do caso 5 obteve um tempo de preenchimento do molde e computacional muito menor do que os outros casos expressos na Tab. 5, corroborando ainda mais a vantagem de utilizar os valores de permeabilidades maiores nas simulações.

Na Fig. 5 é possível visualizar o escoamento da resina dentro do molde na simulação do caso 1, onde a cor vermelha representa a resina e a azul, o ar. 


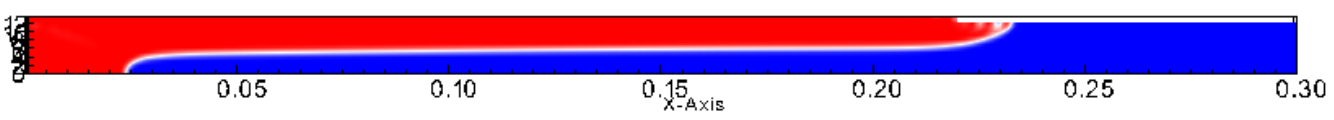

a)

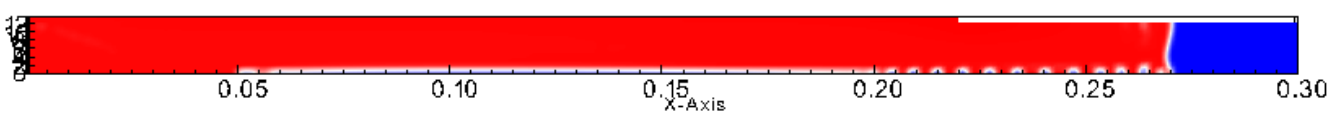

b)

Figura 5. Escoamento da resina dentro do molde. a) no tempo de $3 \mathrm{~s}$. b) no tempo de $29 \mathrm{~s}$.

Para iniciar o processo de otimização da geometria, até o presente momento, considerou-se a relação $K_{x x} / K_{z z}$ igual a um, ou seja, as permeabilidades $K_{x x}$ e $K_{z z}$ são análogas com o valor de $1 \times 10^{-10}$ e as demais propriedades utilizadas nas simulações estão de acordo com a Tab. 1. Nesse sentido, realizou-se cinco simulações em que variou-se o tamanho da malha de fluxo $\left(L_{p}\right)$, cujos valores foram igual à 0,03, 0,06, 0,09, 0,12 e 0,15 m.

Na Fig. 6, pode-se observar as curvas da posição da resina em função do tempo para cada comprimento da malha de fluxo e na Tab. 6 pode ser visualizado a posição e o tempo em que linha de frente de escoamento forma-se retilínea para cada tamanho de $L_{p}$.

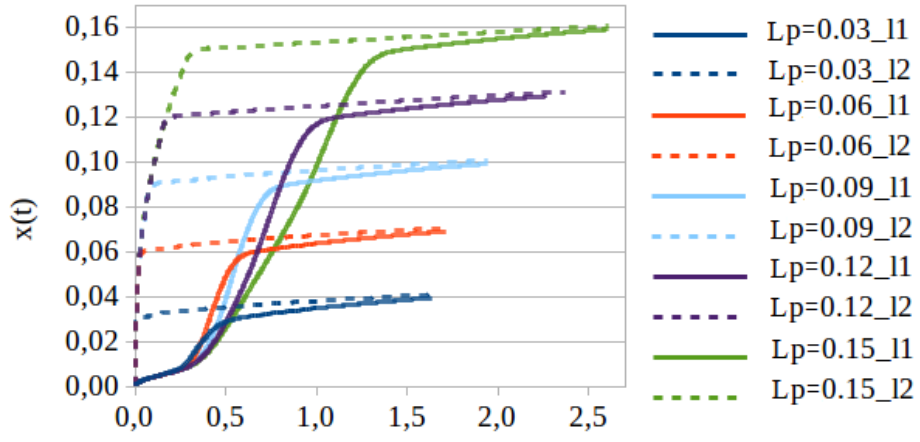

$\mathrm{t}(\mathrm{s})$

Figura 6. Curvas do escoamento da resina para os diferentes comprimentos de $L_{p}$.

Tabela 6. Tempo e posição em que a linha de frente forma-se retilínea.

\begin{tabular}{cccccc}
\hline$L_{p}(m)$ & $K_{\mathrm{xx}} / K_{z z}$ & $t(s)$ & $\begin{array}{c}x(m) \\
\text { Linha 1-11 }\end{array}$ & $\begin{array}{c}x(m) \\
\text { Linha 2-12 }\end{array}$ & $K=x(t)-L_{p}$ \\
\hline 0,03 & 1 & 1,63 & 0,0395262 & 0,0410350 & 0,011035 \\
0,06 & 1 & 1,71 & 0,0692386 & 0,070748 & 0,010747 \\
0,09 & 1 & 1,94 & 0,099301 & 0,1008105 & 0,0108105 \\
0,12 & 1 & 2,26 & 0,1294657 & 0,1309745 & 0,0109745 \\
0,15 & 1 & 2,6 & 0,1592515 & 0,1607603 & 0,0107603 \\
\hline
\end{tabular}

Como pode-se analisar na Tab. 6, para cada comprimento da malha de fluxo considerado nas simulações, a linha de frente de escoamento formou-se em uma posição $x(t)$ distinta, no qual demonstra o tamanho ideal do molde $\left(L_{m}\right)$ para que a resina chegue ao final do molde de forma retilínea e com um tempo total de infiltração menor possível.

A partir desses resultados também, procurou-se obter uma forma de generalizar o tamanho da malha de fluxo para qualquer que seja o comprimento de molde. Na Tab. 6 pode-se visualizar que a diferença entre a posição em que forma-se a linha de frente de escoamento e o comprimento do $L_{p}$ tende a ser uma constante $(K)$. Dessa maneira, é possível obter o tamanho ideal de $L_{p}$ para qualquer que seja o comprimentos de $L_{m}$, por meio da Eq. (8): 


$$
L_{p}=L_{m}-K
$$

onde, $K$ é a média dos valores expressos na Tab. 6.

\section{Considerações finais}

Neste trabalho foi apresentado um estudo numérico sobre a injeção de resina em compósitos espessos nos quais uma malha de fluxo é utilizada para facilitar a injeção de resina. $O$ estudo buscou determinar o tamanho ideal desta malha, em relação ao comprimento total do molde, de maneira que o escoamento ao final do reforço fibroso fosse retilíneo e o tempo de injeção o menor possível. Para tanto, a posição da linha de frente da resina foi rastrea da em dois pontos do molde: próximo as paredes inferior e superior do molde conforme mostrado na Fig. 1. Os resultados mostraram que é possível generalizar o estudo através da apresentação dos resultados para relação $K_{x \chi} / K_{z z}$ e desta forma identificar o comprimento da malha de fluxo $\left(L_{p}\right)$ ideal para qualquer comprimento de molde $\left(L_{m}\right)$.

Agradecimentos: O presente trabalho foi realizado com apoio da Coordenação de Aperfeiçoamento de Pessoal de Nível Superior Brasil (CAPES) - Código de Financiamento 001.

\section{Referências}

1. Brouwer, W. D., Herpet, E. C. F. C., \& Labordus, M. (2003). Vacuum injection moulding for large structural applications. Composites: Part A, 34, 551-558. doi:10.1016/S1359-835X(03)00060-5

2. Correia, N. C., Robitaille, F., Long, A.C, Rudd, C. D., Simácek, P., \& Advani, S.G. (2005). Analysis of the vacuum infusion moulding process: I. Analytical formulation. Composites: Part A, 36, 1645-1656. doi:10.1016/j.compositesa.2005.03.019

3. Gutiérrez, J. C. H., Rubio, J. C. C., Faria, P. E., \& Davim, J. P. (2014). Usinabilidade de Materiais Compósitos Poliméricos para Aplicações Automotivas. Polímeros, 24(6), 711-719. http://dx.doi.org/10.1590/0104-1428.1582

4. Hirt, C. W., \& Nichols, B. D. (1981). Volume of fluid (VOF) method for the dynamics of free boundaries. Journal of Computational Physics, 39(1), 201-225. https://doi.org/10.1016/0021-9991(81)90145-5

5. Hurtado, F. J., Kaiser, A. S., Viedma, A., \& Díaz, S. (2015). Numerical study of the vacum infusion process for laminated composites with diferente fiber orientations. Journal of Reinforced Plastics and Composites, 34(3), 196-212. DOI: $10.1177 / 0731684414566361$

6. Loudad, R., Saouab, A., Beauchene, P., Agogue, R., \& Desjoyeaux, B. (2017). Numerical modeling of vacuum-assisted resin transfer molding using multilayer approach. Journal of Composites Materials, 51(24), 3441-3452. DOI: 10.1177/0021998316687145

7. Magalhães, G. M. C. (2016). Design Construtal e modelagem computacional aplicados à avaliação geométrica de canais abertos em um processo de infusão de resina. Dissertação de Mestrado. https://argo.furg.br/?BDTD11125

8. Srinivasan, V., Salazar, A. J., \& Saito, K. (2011). Modeling the disintegration of modulated liquid jets using volume-of-fluid (VOF) methodology. Applied Mathematical Modeling, 35(8), 3710-3720. https://doi.org/10.1016/j.apm.2011.01.040

9. Wang, P., Drapier, S., Molimard, J., Vautrin, A., \& Minni, J. C. (2011). Numerical and experimental analyses of resin infusion manufacturing processes of composites materials. Journal of Composites Materials, 46(13), 1617-1631. DOI: 10.1177/0021998311421990

10. Yalcinkaya, M. A., \& Sozer, E. M. (2014). Effect of part thickness variation on the mold filling time in vacuum infusion process. Journal of Reinforced Plastics and Composites, 33(23), 2136-2150. DOI:10.1177/0731684414554938 